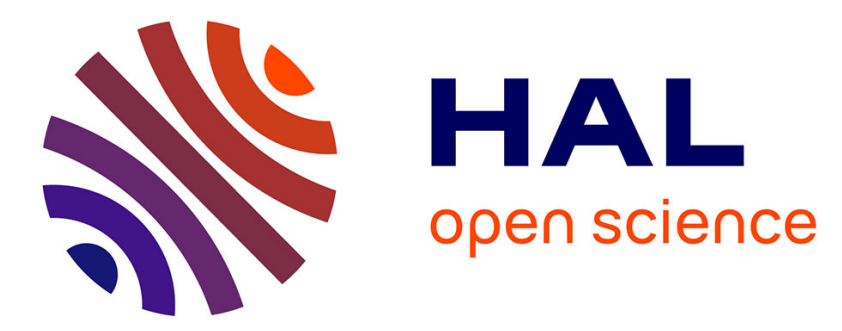

\title{
Analyses multifractales et spatio-temporelles des précipitations du modèle Méso-NH et des données radar
}

Auguste Gires, Ioulia Tchiguirinskaia, D Schertzer, S. Lovejoy

\section{To cite this version:}

Auguste Gires, Ioulia Tchiguirinskaia, D Schertzer, S. Lovejoy. Analyses multifractales et spatiotemporelles des précipitations du modèle Méso-NH et des données radar. Hydrological Sciences Journal, 2011, 56 (3), pp.380-396. 10.1080/02626667.2011.564174 . hal-00677430

\section{HAL Id: hal-00677430 \\ https://hal-enpc.archives-ouvertes.fr/hal-00677430}

Submitted on 24 May 2012

HAL is a multi-disciplinary open access archive for the deposit and dissemination of scientific research documents, whether they are published or not. The documents may come from teaching and research institutions in France or abroad, or from public or private research centers.
L'archive ouverte pluridisciplinaire HAL, est destinée au dépôt et à la diffusion de documents scientifiques de niveau recherche, publiés ou non, émanant des établissements d'enseignement et de recherche français ou étrangers, des laboratoires publics ou privés. 


\title{
Analyses multifractales et spatio-temporelles des précipitations du
}

\section{modèle Méso-NH et des données radar}

\author{
A. Gires ${ }^{1}$, I. Tchiguirinskaia ${ }^{1,2}$, D. Schertzer ${ }^{1,3}$ S. Lovejoy ${ }^{4}$
}

(1) U. Paris-Est, Ecole des Ponts ParisTech, LEESU, Marne-la-Vallee Cedex, France (auguste.gires@leesu.enpc.fr),

(2) CEMAGREF, OHAX, Aix-en-Provence, France,

(3) Meteo-France, CNRM, Paris \&Toulouse, France,

(4) McGill U., Physics dept., Montreal, PQ, Canada

\section{RESUME}

Dans le cadre des multifractals universels, il est possible de caractériser la variabilité spatio-temporelle de la pluie sur une grande gamme d'échelle à l'aide de trois paramètres invariants d'échelles. Dans cette étude, nous avons estimé ces paramètres multifractals sur des simulations numériques effectuées avec le modèle méso-échelle Méso-NH (développé par Météo - France et le Laboratoire d'Aérologie), et des images radar composites, couvrant le même événement pluvieux, à savoir un orage particulièrement violent, dit de type Cévenol, ayant eu lieu sur la partie sud de la France du 5 au 9 septembre 2005. La comparaison des résultats montre que les deux types de données présentent des domaines d'invariance d'échelle relativement similaires, et dont les propriétés sont en accord avec les modèles de précipitation spatio-temporels unifiés et scalant les plus simples. Néanmoins l'évaluation de leurs exposants conduit à des valeurs parfois fortement différentes.

\section{TITLE}

Multifractal and spatio-temporal analysis of the rainfall output of the Méso-NH model and radar data

\section{ABSTRACT}


In the framework of universal multifractals, the spatio-temporal variability of rainfall over a wide range of scales is quantified with the help of three scale invariant parameters. In this paper we estimated these multifractal parameters on numerical simulations performed with the help of Meso-NH (developed by Météo-France and the "Laboratoire d'Aérologie"), and on composite radar images, covering the same heavy rainfall event that occurred on September 5-9, 2005 in the South of France. The comparison of the results shows that both data sets exhibit similar ranges of scaling, whose properties are in agreement with the simplest unified space-time scaling model of rainfall fields. At the same time, some parameter estimates were quite different.

\section{Mots clés}

Précipitation, radar, modèle méso-échelle, invariance d'échelle, multifractal, analyse spatiotemporelle

\section{Key words}

Rainfall, radar, mesoscale model, scaling, multifractal, spatio-temporal analysis

\section{INTRODUCTION}

L'objectif précis de cet article est de procéder à une comparaison multi-échelle des précipitations générées par le modèle MESO-NH avec celles observées par le réseau radar de Météo-France. Cet objectif s'insère dans une question plus large : comment valider un modèle météorologique à l'aide de données qui ne sont pas forcément aux mêmes échelles de temps et d'espace et en évitant la double pénalisation habituelle résultant d'une légère incertitude dans l'espace ou dans le temps ? Cela a conduit à rechercher une méthodologie de validation à travers les échelles spatio-temporelles, et non à une échelle donnée, ce qui correspond à 
comparer la variabilité sur une grande gamme d'échelles du champ simulé et des observations. Si l'extrême variabilité des précipitations sur une grande gamme d'échelle est largement admise, il n'y pas encore un consensus sur la méthodologie pour la quantifier. Ce travail est donc aussi une contribution au développement de cette méthodologie.

Une motivation supplémentaire de ce travail correspond à la question reconnue comme essentielle dans le dernier rapport du GIEC (Solomon et al., 2007) comment évaluer le changement climatique et ses conséquences à des échelles pertinentes pour l'hydrologie, et notamment l'hydrologie urbaine ? Cela répond d'ailleurs à une demande montante des collectivités locales (AFCN, 2008) qui s'interrogent de plus en plus sur les dimensionnements à prévoir de leurs ouvrages, qui correspondent à de longues périodes d'investissement et de durée de vie. La notion de changement climatique ayant déjà fait évoluer les modes de réflexion à ce niveau, il devient donc indispensable d'apporter les outils nécessaires pour y répondre. Notre travail se situe donc pour une part dans le prolongement des travaux menés dans le cadre de ces projets sur des descentes multifractales d'échelles (Biaou, et al., 2005 ; Royer, et al., 2008). Ces descentes d'échelles ont l'originalité d'être à la fois statistiques (comme celles obtenues par des régressions linéaires ou de rang) et à base physique (modèles de cascade), comme nous allons le discuter. Cependant, se posait la question de valider ce type de descente par rapport à des modèles de méso-échelle et/ou des données radars. Cette présente étude est pour une large part un préalable à cette validation.

Ce travail se situe donc de fait à l'intersection de 3 modes d'évaluation/représentation des précipitations, qui ont souvent porté sur des gammes d'échelles différentes : les données radar, les modèles déterministes de météorologie, et les modèles stochastiques de précipitation.

Il convient ici de souligner quelques caractéristiques des modélisations stochastiques des précipitations. Elles se sont au départ contentées de fournir une représentation plus ou 
moins ad hoc des échelles les plus fines des précipitations - hors d'atteinte des modèles déterministes de météorologie - avec les outils mathématiques alors disponibles (processus ponctuels markoviens, [Waymire and Gupta, 1981]). Mais elles se sont progressivement reposées sur des principes de physique de base sur la variabilité qu'elles étaient censées représenter, notamment autour de la question des échelles [Rodriguez-Iturbe, et al., 1984] et enfin de l'invariance d'échelle [Lovejoy and Mandelbrot, 1985 ; Lovejoy and Schertzer 1985 ; Hubert and Carbonnel, 1988 ; Olsson, et al., 1993]. La rupture la plus profonde étant sans doute intervenue avec l'avènement des modèles multifractals de précipitation [Schertzer and Lovejoy, 1987; Gupta and Waymire, 1993; Tessier, et al., 1993 ; Hubert, et al., 1995; Harris, et al., 1996; Marsan, et al., 1996; Olsson and Niemczynowicz, 1996; Bendjoudi, et al., 1997; Deidda, 2000; Desaulnier-Soucy, et al., 2001; Schertzer, et al., 2002; Macor, et al., 2007, Nykanen, 2008]. Elle correspond à relier l'invariance d'échelles à celles des équations génératrices de la pluie, et non seulement à un postulat phénoménologique, et donc à un basculement de modèles linéaires stochastiques (additions de structures de plus en plus petites) vers des modèles fortement non-linéaires (modulation multiplicative des petites structures par les plus grosses). Cette rupture a changé par maints aspects le statut scientifique des précipitations : perçues auparavant comme pouvant faire seulement l'objet de statistiques, elles sont devenues le sujet de débats théoriques, par exemple faut-il [Keddem and Chiu, 1987] ou non [Lovejoy and Schertzer, 1989] traiter les valeurs nulles des précipitations à part, y a-t-il [Schertzer and Lovejoy, 1987, 1997] ou non [Mandelbrot, 1989 ; Gupta and Waymire, 1993] des comportements universels?

Nous commencerons par présenter les données exploitées dans cette étude. Ensuite nous rappellerons les grands principes de l'analyse multifractale qui permet de caractériser la variabilité à travers les échelles à l'aide d'un très petit nombre de paramètres. Enfin, nous 
comparerons les résultats obtenus en analyse spatiale, temporelle et spatio-temporelle avec les deux types de données.

\section{LES DONNEES UTILISEES POUR CETTE ETUDE}

\section{Les simulations Méso-NH}

Le premier type de données utilisées dans cette étude correspond aux précipitations fournies par une simulation numérique effectuée à l'aide du modèle Méso-NH d'un orage Cévenol (intense évènement pluvieux sur le Sud-est de la France, au cours duquel plus de 150 mm d'eau sont tombés en moins de 12h) ayant eut lieu entre le 5 et le 9 septembre 2005 . Méso-NH est un modèle de recherche développé conjointement par Météo France et le Laboratoire d'Aérologie (Univ. P. Sabatier, Toulouse, France) [Lafore, et al., 1998]. MésoNH est un modèle méso-échelle; il permet de traiter des événements météorologiques à l'échelle mésoscopique, qui correspond à une échelle intermédiaire où les distances caractéristiques sont de l'ordre de quelques kilomètres à quelques centaines de kilomètres. C'est un modèle non-hydrostatique, c'est-à-dire que le terme vertical d'accélération n'est pas négligé dans l'équation de conservation de la quantité de mouvement. Cette hypothèse non hydrostatique permet d'être plus proche des équations de Navier-Stokes, et notamment de résoudre la convection profonde. Cependant le modèle est anélastique, ce qui signifie que les ondes acoustiques sont filtrées. La résolution numérique est assurée par un schéma temporel de type leap-frog et un schéma d'advection eulérien. Le modèle a déjà été beaucoup utilisé dans le cadre de recherche à l'échelle mésoscopique (une liste des publications liées à MésoNH est disponible sur le site Internet du modèle : http://mesonh.aero.obs-mip.fr/mesonh/), et sa partie physique a été reprise dans le modèle AROME qui est vient de devenir opérationnel à Météo France. La grille utilisée fait $300 * 300$ mailles dont la taille de chacune est de $0.0215^{\circ}$ (soit environ $2.40 \mathrm{Km}$ ) en latitude et de $0.0284^{\circ}$ (soit environ $3.17 \mathrm{Km}$ ) en longitude. 
Les conditions initiales ainsi que les conditions aux limites ont été obtenues à l'aide d'une simulation numérique effectuée avec le modèle Arpège (Météo France) sur une grille de moins haute résolution dans laquelle était emboîtée la grille exploitée par Méso-NH. Les données sont des précipitations cumulées sur des pas de temps d'échantillonnage de 15 minutes sur chaque pixel -alors que le pas de temps de calcul est de l'ordre de quelques secondes- obtenues à partir de sept simulations de 18 heures démarrant toutes les 12 heures

La figure 1 représente l'évolution du taux moyen de précipitation sur la zone étudiée. L'absence de pluie au début de chaque simulation est due au fait qu'à l'initialisation des simulations les rapports de mélange des hydrométéores (glace, graupel, gouttelette, goutte, grêle, neige) sont tous nuls. L'humidité par contre n'est pas nulle, et va commencer à générer les hydrométéores assez rapidement (quelques pas de temps) si les conditions météorologiques l'imposent, mais il faut cependant un certain temps (quelques heures) pour arriver à des précipitations réalistes. Le manque de réalisme de cette mise en route nous a conduits à ne pas prendre en compte les deux premières heures de chaque simulation dans nos statistiques, i.e. les analyses sont menées sur les 16 dernières heures de chaque simulation

\section{Les données Radar}

Le deuxième type de données utilisées dans cette étude correspond à des mosaïques radar couvrant la France pendant le même évènement. Une mosaïque radar est une image de la réflectivité radar sur toute la France, obtenue par interpolation des réflectivités mesurées par les radars du réseau ARAMIS (Parent du Châtelet, 2005). Une mosaïque correspond à une coupe planaire du champ de précipitation, qui est lui un champ 3D, obtenue par interpolations entre des différentes mesures radar sur un même pixel. La résolution des données est d'un km en espace (en moyenne) et de 15 minutes en temps. 
En plus des problèmes de mesure de la réflectivité comme les échos de sol, la propagation anormale ou la zone de fonte, la conversion de la réflectivité radar $(z)$ en taux de pluie (R) continue de faire débat depuis l'article historique de Marshall et Palmer en 1948 (Marshall and Palmer, 1948) qui a proposé une relation en loi de puissance :

$$
z=a R^{b}
$$

La grille de conversion proposée par Météo-France avec les données et utilisée dans cette étude correspond aux choix des paramètres $a=200$ et $b=1.59$ (z étant exprimé en $\mathrm{mm}^{6} \cdot \mathrm{m}^{-3}$ et $R$ en $\mathrm{mm} \cdot \mathrm{h}^{-1}$ ). La généralisation prochaine des radars bipolarisés doit permettre de calibrer plus ou moins instantanément ces paramètres (Testud et al., 2000, Le Bouar et al., 2001) en estimant ceux de la distribution des tailles de gouttes, à l'aide des réflectivités horizontale et verticale.

Afin de comparer les résultats à ceux des simulations Méso-NH, nous avons sélectionné une zone de $512 \mathrm{~km} * 512 \mathrm{~km}$ couvrant le Sud-Est de la France, et correspondant environ à la zone simulée avec le modèle. L'évolution de l'intensité moyenne de pluie radar sur la zone étudiée est comparée à celle Méso-NH sur la figure 1 . Il y a 2 heures 30 de données manquantes au niveau du deuxième (et principal) pic de précipitations, après un peu plus de 30h. Même si il y a des différences entre les courbes de Méso-NH et les courbes radar, notamment un facteur 1.5 au niveau du premier pic qui de plus n'intervient pas précisément au même instant, ces intensités moyennes présentent des évolutions relativement similaires.

\section{METHODOLOGIE}

Le support de la pluie, c'est à-dire la portion de l'axe du temps ou de l'espace où il pleut, est un objet géométrique dont il est possible d'estimer la dimension fractale, celle d'occurrence de la pluie $\left(D_{F}\right)$ (Hubert 1988). On constate une dépendance de cette dimension fractale par rapport au seuil choisi pour définir le fait qu'il pleuve (Lovejoy et al., 1987, 
Hubert et al., 1995). Lorsque le seuil augmente, le support (et sa dimension fractale) d'occurrence de la pluie diminue. Ainsi le champ de pluie n'est pas caractérisé par une seule dimension fractale, mais par une infinité ; on parle ainsi de champ multifractal. Dans ce cadre la notion de seuil est remplacée par celle invariante d'échelle de singularité. Les propriétés statistiques (moments, probabilité de distribution) d'un champ multifractal sont définies par des lois d'échelle.

On appelle $\mathrm{R}_{\lambda}$ le champ de pluie à la résolution $\lambda(=L / l)$, défini par le rapport entre l'échelle extérieure $L$ et l'échelle d'observation $l$. Numériquement le champ $\mathrm{R}_{\lambda}$ à différentes résolutions $\lambda$ est obtenu en dégradant étape par étape la résolution initiale $\Lambda$ du champ mesurés $R_{\Lambda}$. Une étape de ce processus consiste à diviser la résolution par 2. La valeur affectée à un pixel de résolution $\lambda / 2$ est égale à la moyenne des $2^{d}$ (où $\mathrm{d}$ est la dimension d'étude du champ, dans cette étude : $d=1$ ou 2) pixels de résolution $\lambda$ qui permettent de le faire. Il est usuel de prendre comme statistiques du champ $R_{\lambda}{ }^{q}$ à différentes résolution $\lambda$ ses moments statistiques d'ordre $q<R_{\lambda}^{q}>$. Si le champ étudié est multifractal, alors ces moments sont définies par des lois d'échelle:

$<R_{\lambda}^{q}>\approx \lambda^{K(q)}$

Où $K(q)$ est la fonction d'échelle des moments. Cette fonction est convexe. On peut montrer (Schertzer et Lovejoy , 1987a) que de façon équivalente, les probabilités de dépassement de seuil, définies à partir d'une singularité (arbitaire) $\gamma$, sont reliées par la relation :

$\operatorname{Pr}\left(R_{\lambda} \geq \lambda^{\gamma}\right) \approx \lambda^{-c(\gamma)}$

qui définit la fonction codimension $c(\gamma)$ qui est croissante et convexe. Lorsque $c(\gamma)<d, c(\gamma)$ peut s'interpréter comme la co-dimension fractale du support où la divergence $(\lambda \rightarrow \infty)$ champ est plus rapide que $\lambda^{\gamma}$. Les fonctions $K(\mathrm{q})$ et $c(\gamma)$ sont alors reliées par la transformation de Legendre (Parisi et Frisch, 1985). 
Les fonctions $K(\mathrm{q})$ et $c(\gamma)$ caractérisent la variabilité à travers les échelles du champ étudié et dépendent dans le cas général d'une infinité de paramètres. Dans le cadre des multifractals universels, qui constituent sous des conditions assez générales un attracteur limite pour les cascades multifractales (Schertzer, 1997), ce nombre de paramètre peut être réduit à 3 (Schertzer et Lovejoy, 1987a):

- $H$, le degré de non-conservation, mesure la dépendance en échelle du champ moyen ( $\mathrm{H}=0$ pour un champ conservatif).

- $C_{1}$ mesure l'intermittence moyenne soit le degré de concentration du champ moyen. Plus précisément, $C_{1}$ est la codimension de la singularité moyenne pour un champ conservatif, c'est-à-dire le complément de la dimension fractale $D_{l}$ du support de cette singularité par rapport à la dimension de l'espace physique $d: C_{1}=d$ - $D_{1}$, et donc $C_{1}=0$ pour un champ homogène :

- l'indice de multifractalité $\alpha(0 \leq \alpha \leq 2)$, mesure comment évolue l'intermittence lorsqu'on s'écarte progressivement du champ moyen.

Dans ce cadre l'expression analytique de K(q) est donnée par l'équation suivante :

$$
K(q)=\frac{C_{1}}{\alpha-1}\left(q^{\alpha}-q\right)+H q
$$

Des paramètres $\alpha$ et $C_{1}$ grands reflètent des extrêmes prononcés. Ils sont déterminés à l'aide de la méthode du double moment trace (DTM) (Lavallé et al., 1991). Cette méthode repose sur le fait que, dans le cadre des multifractals universels, la fonction d'échelle des moments $K(q, \eta)$ du champ $R_{\lambda}{ }^{(\eta)}$, obtenu en dégradant la puissance $\eta$ du champ à la résolution maximale (une analyse avec une méthode DTM modifié, Veneziano, 1999, a également été faite mais n'apporte pas de changement dans les estimation qui suivent), s'exprime aisément en fonction de $\alpha$ :

$$
\left\langle\left(R_{\lambda}{ }^{(\eta)}\right)^{q}\right\rangle \approx \lambda^{K(q, \eta)}=\lambda^{\eta^{\alpha} K(q)}
$$


Ainsi $\alpha$ est la pente de la partie linéaire de $K(q, \eta)$ en fonction de $\eta$ dans un graphique loglog. Dans la suite, la portion de la courbe utilisée pour estimer $\alpha$ est représentée en trait plein sur les courbes de détermination de $\alpha$.

La première étape d'une analyse multifractale consiste souvent en une analyse spectrale. La présence d'une pente spectrale $\beta$ relativement constante sur une gamme de fréquences $f$ $\left(\mathrm{E}(f)=f^{\beta}\right)$ correspond à une première confirmation du comportement scalant du champ étudié. Le degré de non conservation $H$ se déduit de la pente spectrale $\beta$, par la relation $\beta=1+2 H-K(2 a)$ (Yaglom, 1966, Tessier et al., 1993). Une interprétation du «a » est qu'il existe un champ conservatif $\varepsilon_{\lambda}$ tel que $R_{\lambda} \propto \varepsilon_{\lambda}{ }^{a} \lambda^{-H}$. Dans cette étude l'approximation classique de a par 1 est utilisée.

Les données spatio-temporelles exploitées, à savoir la pluie en un certain nombre de pixels d'une surface donnée pendant un certain nombre de pas de temps, permettent de mener trois types d'analyses :

- des analyses spatiales : elles consistent à faire, pour chaque pas de temps, l'analyse spatiale 2D du champ de pluie.

- des analyses temporelles : elles consistent à faire, pour chaque pixel, l'analyse de la série temporelle 1D pluviométrique.

- des analyses spatio-temporelles : elles consistent à faire directement l'analyse 1+2 D du champ de pluie.

Pour les trois types d'études, nous avons effectué des analyses échantillon par échantillon (c'est-à-dire pas de temps par pas de temps en analyse spatiale et pixels par pixels en analyse temporelle) et en moyenne d'ensemble. Dans ce cas, chaque échantillon est considéré comme une réalisation du phénomène. La moyenne faite dans les équations 2 et 5 porte alors sur tous les échantillons dégradés indépendamment et non plus sur un seul. Les équations 2 et 5 ne sont à priori valables qu'en moyenne sur un ensemble infini de réalisation. 
Il existe cependant des propriétés plus fortes qui assurent une convergence rapide. Ainsi les analyses «échantillon par échantillon» sont moins robustes que celles en «moyenne d'ensemble » qui prennent en compte d'avantage d'échantillon, mais elles permettent tout de même de mettre en lumière certains comportements en terme d'évolution temporelle ou de répartition spatiale des paramètres. Ayant des données manquantes pour les données radar

pour la période de temps correspondant à la période de la $3^{\text {ème }}$ simulation, et voulant pouvoir comparer les résultats entre les deux types de données, nous n'avons pas fait porter la moyenne d'ensemble sur cette période, bien qu'elle corresponde au pic des précipitations.

\section{ANALYSE SPATIALE}

\section{ANALYSE DU COMPORTEMENT SCALANT}

La figure 2 présente le spectre moyen pour les simulations Méso-NH, et la même grandeur pour les données radar. La présence d'une pente spectrale donne une première confirmation du comportement scalant de la pluie. Les deux types de données semblent cependant présenter une rupture de pente. Pour les données radar, cette rupture aurait lieu entre les nombres d'onde 25 et 35 , soit pour des distances comprises entre 14 et $20 \mathrm{~km}$. Pour les simulations Méso-NH, cette rupture est moins évidente et en ajustant comme pour les données radar deux droites de régression linéaire, elle aurait lieu pour des nombres d'onde compris entre 15 et 25 , ce qui correspondrait à une rupture en espace comprise entre 30 et 50 km. Il serait même possible de distinguer une deuxième rupture aux alentours du nombre d'onde 80. Quels que soit le type de données et la gamme d'échelle considérée, les pentes spectrales obtenues sont inférieures à la dimension du champ (égale à 2 dans cette analyse spatiale), ce qui signifie que la méthode DTM peut s'appliquer directement au champ de pluie. Dans le cas contraire, il aurait fallu faire une intégration fractionnaire du champ, ou bien analyser les fluctuations (module du gradient) de ce dernier (Nykanen, 2008). La figure 3 
illustre la première étape de la méthode DTM $(q=1.5, \eta=0.66 ; 1 ; 1.52 ; 2.31)$, à savoir la vérification du comportement scalant à travers un graphique log-log du double moment trace en fonction de la résolution. Le fait que les coefficients $R^{2}$ de détermination des régressions linéaires deviennent meilleurs, notamment pour les grandes échelles, en considérant une rupture, tend à confirmer la présence de cette rupture de pente et la nécessité de la prendre en compte. Cette rupture à lieu pour la résolution $\lambda=32$ (soit $25 \mathrm{Km}$ en latitude et $19 \mathrm{Km}$ en longitude) pour les simulations Méso-NH et pour $\lambda=32$ (soit $16 \mathrm{Km}$ ) pour les données radar. Ceci confirme les résultats de l'analyse spectrale. La présence de cette rupture sur les deux types de données à des échelles spatiales comparables tend à montrer qu'il ne s'agit pas d'un artefact, mais probablement d'un phénomène physique ou d'échantillonnage. Cette dernière cause est possible du fait que nous disposons seulement des hauteurs de pluie cumulées sur 15 minutes, ce qui est assez long par rapport à la résolution spatiale de l'ordre du kilomètre. Cette interprétation serait en accord avec le constat que le scaling est en moyenne légèrement meilleur (3\%) pour les échelles supérieures à $20 \mathrm{~km}$ que pour les échelles plus petites. Ainsi l'analyse de ces petites échelles est certainement plus complexe à mener avec les données dont nous disposons, mais sont d'autant moins négligeables que Zawadzki (1987) estimait à 20 minutes le temps d'auto-corrélation des orages. Une explication de cette rupture à travers la topographie est a priori exclue, dans la mesure où une analyse multifracal menée sur les données de relief ne met pas en évidence de rupture, au moins entre $2-3 \mathrm{~km}$ et $150-200 \mathrm{~km}$.

\section{ANALYSE ECHANTILLON PAR ECHANTILLON}

L'évolution temporelle des différents paramètres en grandes et petites échelles pour les simulations Méso-NH et pour les données radar est représentée sur la figure 4. Le tableau 1 résume les statistiques classiques des estimations de ces paramètres (moyenne, écart-type et coefficient de variations). Il convient de considérer avec prudence ces statistiques pour les 
simulations Méso-NH, car elles ont été calculées sur les 64 derniers pas de temps de chaque simulation (pour éviter les problèmes d'initialisation du modèle), si bien qu'il reste un intervalle de recouvrement entre deux simulations successives de $4 \mathrm{~h}$ et les pas de temps correspondant interviennent donc deux fois, mais pour deux simulations différentes, ce qui peut légèrement fausser les résultats (en donnant d'une certaine façon un poids double à ces périodes de recouvrement). Une partie de la fluctuation des paramètres est expliquée par les incertitudes sur l'estimation de ces derniers. En effet, en travaillant pas de temps par pas de temps, le nombre de pixels est assez faible notamment en grandes échelles, si bien que les statistiques obtenues ne sont pas très fiables. On constate néanmoins que ces grandeurs fluctuent plus lors de la période de moindre pluie entre la $45^{\text {ème }}$ et la $60^{\text {ème }}$ heure. Ceci est probablement causé par une influence plus grande des effets de quantification qui conduit à des incertitudes de détermination plus grandes des paramètres multifractals. Il convient également de noter que les estimations de $\alpha$ pour le modèle Méso-NH ont tendance, principalement en grandes échelles, à augmenter significativement au cours de chaque période de simulation après une période de relative stabilité d'un peu moins de 10 heures, sans que l'on observe cette tendance sur les données radar des périodes correspondantes. C'est particulièrement visible sur les 3 premières et l'avant dernière périodes de simulation, qui sont les périodes de plus forte pluie. Cette augmentation d' $\alpha$ est assez gênante puisque elle suggère que la variabilité spatiale de la pluie simulée par le modèle Méso-NH a du mal à se stabiliser indépendamment de la durée de simulation du modèle. Mentionnons tout de même qu'en présence de nombreux pixels sans pluie (comme c'est le cas au début de chaque période de simulation Méso-NH), l'estimation de $\alpha$ par la méthode DTM peut présenter certains biais qui engendrent une sous-estimation de $\alpha$. Des éléments d'explication sont fournis dans la partie sur l'analyse temporelle. Signalons pour finir que les valeurs moyennes de $H \neq 0$ pour les deux types de données témoignent d'un champ légèrement non conservatif. 


\section{ANALYSE EN MOYENNE D'ENSEMBLE}

Afin d'avoir des estimations des paramètres multifractals plus fiables, nous avons mis en œuvre la méthode DTM en prenant en compte des moyennes d'ensemble sur les différents pas de temps. Pour les données Méso-NH, les pas de temps pris en compte correspondent aux 64 derniers de chaque simulation, ce qui signifie que les pas de temps simulés deux fois (il reste 4 h de recouvrement entre deux simulations successives), sont d'une certaine façon pris en compte deux fois (une fois dans chaque simulation). Afin de pouvoir comparer sans biais les résultats entre les données radar et Méso-NH et avec l'analyse temporelle menée après, les mêmes pas de temps (avec un poids double pour certains) ont été pris en compte pour les données radar. La figure 2, déjà discutée, représente la première étape de la méthode à savoir l'estimation de $K(q, \eta)$. Les courbes de détermination de $\alpha$ sont sur la figure 5. Les principaux résultats obtenus sont dans le tableau 2. Les valeurs de $\alpha$ et $C_{1}$ sont différentes entre les simulations Méso-NH et les données radar. En effet, $\alpha$ a tendance à être plus grand et $C_{1}$ plus petit pour les données radar que pour les simulations. Ce constat rend assez complexe l'interprétation en termes d'extrême. Il signifie néanmoins que la variabilité de la pluie serait sous-représentée dans les simulations, mais que son intermittence moyenne serait sur-évaluée. Il est possible de raffiner l'analyse en regardant la singularité maximale observable $\gamma_{s}$ (Hubert, 1993, Royer, 2008), qui est une grandeur invariante d'échelle. Cette dernière est définie comme la singularité pour laquelle la codimension fractale du support $c\left(\gamma_{s}\right)$ devient égale à la dimension du champ $d$ (dans l'analyse spatiale $d=2$ ). Pour les grandes échelles, on constate que malgré les différences de valeurs entre les exposants multifractals, les singularités maximales observables restent comparables. 
Evoquons maintenant les résultats pour les petites échelles, dont on rappelle que l'analyse du comportement scalant est plus complexe du fait que la résolution temporelle de 15 minutes n'est pas vraiment adaptée à la résolution spatiale de l'ordre du kilomètre. Pour les deux types de données, on retrouve des différences similaires de comportement entre les gammes d'échelle : $\alpha$ est plus grand alors que $C_{1}$ et la singularité maximale observable $\gamma_{s}$ sont plus petits pour les petites échelles que pour les grandes échelles. L'estimation de la singularité maximale observable en petites échelles différent en fonction du type de données. Elle vaut 0.60 et 0.84 respectivement pour Méso-NH et pour les données radar. Ceci signifie que le modèle a tendance à sous-estimer les extrêmes sur cette gamme d'échelle. Le paramètre $\alpha>1$ obtenu quel soit le type de données pour les petites échelles évoque le comportement non-classique « self-organised critical » (Bak et al., 1988) qui implique que les singularités ne sont plus bornées. Cette différence profonde de comportement entre les gammes d'échelles implique que les statistiques à petites échelles ne peuvent pas toujours être déduites des statistiques obtenues à grandes échelles. Il convient d'ajouter que les valeurs des paramètres multifractals estimées pour le radar $\left(\alpha \approx 1.6, C_{1} \approx 0.16\right)$ sont en accord avec celles décrivant habituellement la turbulence (Schmitt, 1992, Lazarev, 1994), ce qui attesterait que la pluie se comporte comme un scalaire passif aux petites échelles.

Afin de confirmer ces résultats, il faudrait mener ce type d'analyse sur des données possédant une résolution temporelle bien meilleure.

\section{ANALYSE TEMPORELLE}

Nous rappelons que les données Méso-NH correspondent à la pluie accumulée toutes les 15 minutes et qu'elles ne sont pas issues d'une seule simulation durant 4 jours, mais de 7 
simulations de $18 \mathrm{~h}$ et démarrant toutes les 12 heures avec des conditions initiales réactualisées. Ainsi les périodes sur lesquelles portent deux simulations successives se superposent pendant 6 heures. Tenant compte qu'il est relativement plus aisé, mais non indispensable, d'agréger les données par une puissance de 2, nous avons analysé les 64 derniers pas de temps d'échantillonnage (de 15 minutes) de chaque simulation. Afin de pouvoir comparer les résultats nous avons pour chaque simulation procédé aux mêmes analyses sur les pas de temps correspondant pour les données radar. Comme précédemment mentionné, nous n'avons pas analysé les pas de temps correspondant à la $3^{\text {ème }}$ simulation Méso-NH car il y avait de nombreuses données radar manquantes à cette période. Nous rappelons que par analyse temporelle, nous entendons l'analyse de la série temporelle de pluie en chaque pixel du modèle Méso-NH ou de l'image radar.

\section{ANALYSE DU COMPORTEMENT SCALANT}

Afin de vérifier le comportement scalant du champ étudié, nous avons travaillé en moyenne d'ensemble pour obtenir les courbes de loi d'échelle. Il ressort de l'analyse spectrale (Figure 6) qu'aussi bien pour les données Méso-NH que pour les données radar le comportement scalant présente une rupture de comportement aux alentours de $1 \mathrm{~h}$ (correspondant à un nombre d'onde d'environ 15). Pour les données Méso-NH la pente spectrale est supérieure en petites échelles à celle en grandes échelles. Le phénomène inverse est observé pour les données radar. Cette rupture est moins visible sur les courbes de détermination de loi d'échelle (figure 7), mais par cohérence avec l'analyse spectrale, les gammes d'échelles $1 \mathrm{~h}-16 \mathrm{~h}$ et $15 \mathrm{~min}-1 \mathrm{~h}$ ont tout de même été étudiées indépendamment. Ceci permet d'améliorer légèrement les coefficients de détermination des courbes. Les résultats de l'analyse DTM en petite échelle ayant été obtenus avec une gamme d'échelle réduite 
(seulement 3 points) ne sont pas très fiables, et ne seront par conséquent que très brièvement évoqués.

En tenant compte des ruptures spatiale $(20 \mathrm{Km})$ et temporelle $(1 \mathrm{~h})$, il est possible de définir une vitesse «caractéristique » de l'événement de l'ordre de $6 \mathrm{~m} / \mathrm{s}$. Cette grandeur est en accord avec la vitesse caractéristique classique du vent de $10 \mathrm{~m} / \mathrm{s}$, et correspond à un vent de force 3 sur l'échelle de Beaufort (pour un point de vue multifractal sur l'échelle de Beaufort, Kerman, 1993). Sans prendre en compte de rupture temporelle, on obtient une vitesse caractéristique de l'ordre de $24 \mathrm{~m} / \mathrm{s}$, soit un vent de force 9 , ce qui est très élevé.

\section{ANALYSE ECHANTILLON PAR ECHANTILLON}

La figure 8 présente les résultats pour la dernière période de simulations étudiées pour les grandes échelles. Les phénomènes observés sont globalement retrouvés pour toutes les périodes de simulation. Le tableau 3 récapitule les principales statistiques des paramètres en grandes échelles pour cette période, dont la corrélation avec la codimension -fractale- du support de la pluie $C_{\mathrm{F}}\left(C_{F}=1-D_{F}\right.$, où $D_{F}$ est la dimension fractale du support de la pluie calculée sur l'ensemble des pixels). Comme nous le discuterons au prochain paragraphe, une trop grande codimension du support de la pluie introduit un biais dans l'estimation des paramètres multifractals. En ce qui concerne $C_{1}$, on observe une corrélation entre son estimation et la codimension du support, ce qui correspond à une anti-corrélation avec l'intensité. Cette corrélation est plus prononcée pour les données radar que pour les données Méso-NH. Pour $\alpha$, les données radar sont fortement anti-corrélées avec la codimension du support, tandis que c'est nettement moins vrai pour les données Méso-NH. On retrouve ce comportement pour toutes les périodes avec les données radar, et de façon moins prononcée avec les données Méso-NH. Ainsi, les données radar présentent un comportement plus cohérent dans le temps que les données Méso-NH. Compte tenu des différences importantes 
entre les simulations, et des forts écart-type qu'il y a au sein d'une même période, les estimations moyennes de $\alpha$ et $C_{1}$ sont probablement entachées d'une large incertitude et donc difficilement exploitables.

Les disparités trouvées dans l'estimation de $\alpha$ et $C_{1}$ peuvent résulter de causes physiques et/ou d'incertitude dans l'estimation des paramètres. Avant de poursuivre, il convient d'expliquer la présence des paliers inférieur et supérieur sur la courbe d'estimation de $\alpha$ dans la méthode DTM (équation 5 et figure 7). Hittinger (2008) a montré que les «transitions de phase multifractales de second ordre » (Schertzer, 1992), induites par la taille finie de l'échantillon, font que l'estimation empirique de $K(q, \eta)$, pour un ordre $q(>1)$ donné et sur un nombre d'échantillons proche de l'unité, devient une constante,

$$
K(q, \eta)=(q-1) d
$$

au lieu de la loi de puissance théorique, à partir d'un ordre critique $\eta_{+}$, défini par l'équation :

$q \eta_{+}(q)=\left(d / C_{1}\right)^{1 / \alpha}$

où $d$ est la dimension de l'espace (i.e., $d=1$ dans le cas de séries temporelles). Cela correspond au palier supérieur que l'on peut observer sur la figure 7.

Le palier inférieur peut être expliqué par la présence de nombreux zéros dans les échantillons étudiés. En effet, ces derniers peuvent correspondre au fait qu'il y a une singularité minimale $\left(\gamma_{\min }\right)$, en dessous de laquelle la pluie n'est pas observée (limite de la dynamique du capteur) ou non enregistrée (problème de quantification) ou encore non simulée (limite de représentation numérique des nombres : « underflow »). Dans ce cas, la fonction codimension $c(\gamma)$ devient pour $\gamma<\gamma_{\min }$, une constante $c_{\min }$, la codimension (fractale) du support de la pluie. Par la transformée de Legendre, la fonction d'échelle des moments est linéaire pour des faibles valeurs de $q$ (de Lima, 1999). Ainsi K(q,ך) est une constante 
$K(q, \eta)=(q-1) c_{\min }$

jusqu'à l'ordre critique défini par l'équation :

$\eta_{-}(q)=\left(c_{\min } / C_{1}\right)^{1 / \alpha}$

Ainsi pour des fortes valeurs de la codimension $\mathrm{c}_{\min }$ du support de la pluie, la zone linéaire de $\mathrm{K}(\mathrm{q}, \eta)$ dans un graphique $\log$-log, qui permet d'estimer $\alpha$, se trouvera fortement réduite par ces deux paliers horizontaux correspondant à $\left\{\eta \leq \eta_{-}\right.$(q) $\}$et $\left\{\eta \geq \eta_{+}\right.$(q) $\}$, qui de plus introduisent des courbures transitoires. Ces deux effets conduisent à des sous-estimations artificielles de la pente $\alpha$.

\section{ANALYSE EN MOYENNE D'ENSEMBLE}

Afin d'avoir des estimations plus fiables, et que l'on puisse comparer à celles obtenues dans l'analyse spatiale, nous avons mis en œuvre la méthode DTM en faisant des moyennes d'ensemble sur un grand nombre d'échantillon (tous les pixels des différentes simulations). La première étape de la méthode DTM est présentée figure 7, et a déjà été discutée. Les courbes de détermination de $\alpha$ sont présentées figure 9. Les valeurs trouvées sont présentées dans le tableau 4. En grandes échelles, par rapport à l'analyse spatiale, on retrouve pour chaque type de données, les mêmes valeurs de $\alpha$ (0.54 pour Méso-NH et 0.82 pour radar) et des valeurs de $C_{1}(0.50$ pour Méso-NH et 0.35 pour radar) plus petites. L'interprétation de ces résultats (constance de $\alpha$, variation de $C_{1}$ ) requière de se placer dans le cadre d'une analyse spatiotemporelle, qui est introduite dans la section suivante. On peut cependant déjà noter que Meso-NH semble sous-estimer la variabilité de la pluie et par contre surestimer légèrement son intermittence moyenne. Concernant les petites échelles, dont on rappelle que les résultats sont peu fiables compte tenu de la gamme d'échelle réduites disponible, on peut simplement noter que comme dans l'analyse spatiale, $\alpha$ est plus grand et $C_{1}$ plus petit que pour les grandes échelles. 


\section{ANALYSE SPATIO-TEMPORELLE}

Les modèles spatio-temporels unifiés et scalant les plus simples de pluie (Marsan, 1996, Deidda, 2000, Macor, 2007, Radkevich, 2008) se basent sur l'hypothèse d'un exposant d'anisotropie entre l'espace et le temps. Ainsi, ils suggèrent que les fonctions d'échelle des moments spatiales et temporelles devraient être proportionnelles, c'est-à-dire

$$
K_{\text {espace }}(q)=\frac{K_{\text {temps }}(q)}{1-H_{t}}
$$

Où $H_{t}$ est l'exposant d'anisotropie entre l'espace et le temps. Ceci implique des $\alpha$ identiques et des $C_{1}$ et $\mathrm{H}$ présentant le même rapport.

$$
\frac{C_{1, \text { espace }}}{C_{1, \text { temps }}}=\frac{H_{\text {espace }}}{H_{\text {temps }}}=\frac{1}{1-H_{t}}(10)
$$

Les résultats trouvés dans les analyses temporelles et spatiales aux grandes échelles sont globalement en accord avec ce cadre théorique, notamment pour les données radar. En effet, pour ces dernières le rapport $C_{1, \text { espace }} / C_{1 \text {,temps }}$ obtenu correspond à un exposant d'anisotropie égale à 0.22 , tandis que le rapport $H_{\text {espace }} / H_{\text {temps }}$ mène à un $H_{t}$ de 0.38 . Ces grandeurs sont comparables et compatibles avec la valeur de $1 / 3$ qui correspondrait à la théorie de Kolmogorov (Kolmogorov, 1962, Marsan, 1996). Pour les données Méso-NH, le rapport entre les $C_{1}$ mène à un $H_{t}$ de 0.30 , ce qui en accord avec les données radar. En revanche le rapport des $H$ ne permet pas de rentrer dans ce cadre théorique puisqu'il donne un $H_{t}$ de -0.01 . Ainsi, on obtient pour les grandes échelles un bon accord des données radar avec le modèle spatiotemporel unifié, mais ce n'est pas le cas pour l'ensemble des paramètres des simulations Méso-NH. 
Afin de confirmer ces résultats, des analyses spatio-temporelles ont été faites. Dans ces dernières les dimensions spatiales et temporelles sont prises en compte simultanément. Nous avons considéré une isotropie parfaite entre les deux directions d'espace, et un exposant d'anisotropie espace-temps égal à 1/3 (qui correspondrait à la théorie de Kolmogorov, et qui a été à peu près retrouvé dans les analyses spatiale et temporelle des données). Ainsi dans le DTM, lors de dégradation du champ, lorsque chaque dimension d'espace est divisé par $\lambda$, la dimension de temps, est divisée par $\lambda^{1-1 / 3}$. Ici nous avons choisi $\lambda$ égal à 3 , si bien que la dimension temporelle est divisée par $2\left(3^{2 / 3} \approx 2.08\right)$. Pour les données Méso-NH, nous avons considéré des échantillons de $3^{5} * 3^{5} * 2^{5}=243 * 243 * 32$ pixels. Pour les données radar, nous nous sommes ramené à des échantillons de cette taille en dégradant préalablement les deux dimensions d'espace d'un facteur 2. Nous avons travaillé en moyenne d'ensemble avec les échantillons disponibles pour les deux types de données.

La première étape du DTM, qui permet de vérifier le comportement scalant du champ, est présentée figure 10 . Une rupture a été prise en compte en $\lambda=27$, ce qui correspond à environ $20 \mathrm{Km}$ en espace et $4 \mathrm{~h}$ en temps. Cette rupture du comportement scalant est équivalente à celles déjà observée dans les analyses spatiales et temporelles.

Les courbes de détermination de $\alpha$ pour les grandes échelles sont en figure 11 . Le tableau 5 fourni les estimations obtenues des paramètres multifractals pour les deux types de données en petites échelles et en grandes échelles. En ce qui concerne les données radar, pour les grandes échelles, nous retrouvons les mêmes valeurs des paramètres que dans l'analyse spatiale, ce qui est parfaitement cohérent avec le modèle spatio-temporelle unifié précédemment évoqué. Pour les données Méso-NH, la courbe de détermination de $\alpha$ est nettement moins lisse que pour les données radar et perd même sa convexité, ce qui entraine des estimations des paramètres moins fiables. On obtient un $\alpha$ plus grand que dans l'analyse 
spatiale, et un $C_{1}$ nettement plus petit, compris entre celui de l'analyse spatiale et de l'analyse temporelle. Pour les petites échelles, les estimations se rapprochent de celles trouvées dans l'analyse spatiale. Ce résultat est à prendre avec prudence dans la mesure où la résolution temporelle initiale des données, qui est trop faible, y joue un rôle important.

\section{CONCLUSION}

Dans cette étude, nous avons procédé à une analyse multifractale de la variabilité spatio-temporelle de la pluie. Dans le cadre des multifractals universels utilisés, cette variabilité est quantifiée à l'aide de simplement trois paramètres caractérisant les lois d'échelle reliant les statistiques du champ de pluie à différentes résolutions.

L'évènement pluvieux que nous avons étudié est un orage de type Cévenol qui a eu lieu sur la partie Sud - Est de la France du 5 au 9 septembre 2005. Nous avons comparé les résultats obtenus à partir de simulations numériques faites avec le modèle météorologique méso-échelle non hydrostatique Méso-NH, et des données radar couvrant cet événement météorologique.

Il ressort de l'analyse des données radar des conclusions qui constituent une étape importante en vue de la validation des descentes d'échelle spatio-temporelles et des simulations multifractales des champs de pluie. En effet le comportement observé présente les deux caractéristiques suivantes :

- Une rupture de comportement scalant dans l'analyse spatiale à une échelle d'espace d'environ $20 \mathrm{Km}$. L'origine de cette dernière est physique ou bien lié au pas de temps d'échantillonnage de la mesure. En effet la résolution temporelle (15min) des données est trop faible par rapport à la résolution spatiale qui est de l'ordre du kilomètre. Une rupture de comportement est également trouvée à environ 1 heure dans l'analyse temporelle. 
- La comparaison des estimations des paramètres multifractals obtenues aux grandes échelles dans les analyses spatiales et temporelles est en très bon accord avec les prédictions des modèles de précipitation spatio-temporels unifiés et scalant les plus simples, qui reposent sur un exposant d'anisotropie entre les dimensions spatiales et temporelles. Ce résultat est confirmé par l'analyse spatio-temporelle directe du champ de pluie.

En ce qui concerne les simulations Méso-NH, on retrouve des ruptures similaires de comportement dans les analyses spatiales et temporelles. Certains résultats sont en accord avec le modèle spatio-temporelle unifié, mais il convient de noter que l'accord est moins bon que pour les données radar, particulièrement au niveau du degré de non conservation du champ. En général, les estimations numériques des paramètres multifractals présentent des différences non négligeables avec celles obtenues sur les données radar : la variabilité de la pluie est sous-représentée par le modèle Méso-NH, tandis que l'intermittence moyenne est sur-représentée.

Afin de confirmer ces résultats, il convient de mener ces comparaisons sur d'autres évènements pluvieux, avec des données dont la résolution temporelle serait plus grande. Il convient aussi de développer des techniques d'estimations des paramètres multifractals robustes à la présence de nombreux zéros, qui comme nous l'avons vu peuvent introduire des biais dans les méthodes classiques.

\section{REMERCIEMENTS}

Les auteurs remercient Météo-France pour l'accès aux données de simulation Méso-NH, des données radar dans le cadre du projet MHYM (Multiplicité d'Échelles en Météorologie et Hydrologie, http://www.enpc.fr/multifractal/projects/MHYM/) de collaboration scientifique entre l'Ecole des Ponts ParisTech et Météo-France. Ils remercient particulièrement Christine 
Lac, Véronique Ducroq, Jean-Michel Veisseire, Jacques Parent du Châtelet, Jean-François Royer pour leurs suggestions dans l'exploitation de ces données et leurs commentaires avisés.

Cette étude a été effectuée pour une part dans le cadre du projet GARP-3C du programme R2DS. Les auteurs remercient également l'école doctorale Sciences Ingénierie et Environnement pour son soutien. Les auteurs remercient également les deux relecteurs anonymes dont les suggestions et commentaires ont permis d'améliorer la qualité de ce manuscrit.

\section{BIBLIOGRAPHIE}

AFCN (Ed.) (2008), Adaptation au changement climatique: les risques naturels, Association Française pour la Prévention des Catastrophes Naturelles (AFPCN).

Bak, P., C. Tang, and K. Weissenfeld, 1988, Self-Organized Criticality, Phys. Rev. Lett A38, 364-374

Bendjoudi, H., Hubert, P., Schertzer, D., Lovejoy, S. (1997), Interprétation multifractale des courbes intensité-durée-fréquence des précipitations, Multifractal point of view on rainfall intensity-duration-frequency curves, Comptes Rendus de l'Académie des Sciences - Series IIA - Earth and Planetary Science, 325, 323-326.

Biaou A., Chauvin F., Royer J-F., Schertzer D. (2005) : Analyse multifractale des précipitations dans un scénario GIEC du CNRM. Note de centre GMGEC, CNRM, 101, 45 pp

Deidda, R. (2000), Rainfall downscaling in a space-time multifractal framework, Water Resour. Res., 36, 1779_1794.

Desaulnier-Soucy, N., Lovejoy, S., Schertzer, D. (2001), The continuum limit in rain and the HYDROP experiment, J. Atm. Res, 59-60, 163-197.

De Lima M.I.P (1998), Multifractals and the temporal structure of rainfall, $\mathrm{PhD}$ thesis, Wageningen Agricultutal University, 229pp

De Lima, M.I.P., and Grasman, J. (1999), Multifractal analysis of 15-min and daily rainfall from a semi-arid region in Portugal, Journal of Hydrology 220, 1-11

Gupta, V. K., and E. Waymire (1993), A Statistical Analysis of Mesoscale Rainfall as a Random Cascade, J. Appl. Meteor., 32, 251-267.

Harris, D., Menabde, M, Seed, A., Austin, G. (1996), Multifractal characterization of rain fields with a strong orographic influence, J. Geophys. Res., 101, 26405-26414. 
Hubert, P., and J. P. Carbonnel (1988), Caractérisation fractale de la variabilité et de l'anisotropie des précipitations tropicales, C. R. Acad. Sci. Paris, 2, 909-914.

Hubert, P., Tessier, Y., Ladoy, P., Lovejoy, S., Schertzer, D., Carbonnel, J.P, Violette, S., Desurosne, I., Schmitt, F., (1993), Multifractals and extreme rainfall events, Geophys. Res. Lett., 20, 931-934

Hubert, P., Friggit F., Carbonnel J.P. (1995), Multifractal structure of rainfall occurrence in west Africa, edited by Z. W. Kundzewicz, pp. 109-113., Cambridge University Press, Cambridge.

Keddem, B., and L. S. Chiu (1987), Are rain rate processes self-similar?, Wat. Resour. Res., 23, 1816-1818.

Kerman, B. R. (1993), A multifractal equivalent of the Beaufort scale for sea-state, Geophys. Res. Lett., 20(4), 297-300

Kolmogorov, A.N. (1962), A refinement of previous hypotheses concerning the local structure of turbulence in viscous incompressible fluid at high Reynolds number, J. Fluid. Mech., 83 (349)

Lafore, J.-P., J. Stein, N. Asencio, P. Bougeault, V. Ducrocq, J. Duron, C. Fischer, P. Héreil, P. Mascart, V. Masson, J.-P. Pinty, J.-L. Redelsperger, E. Richard, and J. Vilà-Guerau de Arellano (1998), The Meso-NH Atmospheric Simulation System. Part I: adiabatic formulation and control simulations. Scientific objectives and experimental design, Ann. Geophys., 16, 90109

Lavallée, D. (1991), Multifractal techniques: analysis and simulation of turbulent fields, $P h D$ Thesis, McGill University, Montréal (Québec), Canada.

Lavallée, D., Lovejoy, S., Ladoy, P. (1991a), Nonlinear variability and landscape topography: analysis and simulation, Fractas in geography, L. De Cola et N. Lam (éditeurs). PrenticeHall, 171-205

Lavallee, D., Schertzer, D., Lovejoy, S. (1991b), On the determination of the codimension function, Non-linear variability in geophysics, Kluwer, Dordrecht, 99-110

Lavallée, D., S. Lovejoy, D. Schertzer, P. Ladoy, (1993), Nonlinear variability and Landscape topography: analysis and simulation. Fractals in Geography, Eds. L. De Cola, N. Lam, 158192, PTR, Prentice Hall.

Lazarev, A., D. Schertzer, S. Lovejoy, Y. Chigirinskaya, 1994, Unified multifractal atmospheric dynamics tested in the tropics: part II, vertical scaling and Generalized Scale Invariance, Nonlinear Processes in Geophysics, 1, 115-123.

Le Bouar E., Testud J., Keenan T.D (2001), Validation of the rain profiling aglorithn « ZPHI » from the C-band polarimetric weather radar in Darwin. J Atmos. Oceanic Tech., 18, $1819-1837$ 
Lovejoy, S., and B. B. Mandelbrot (1985), Fractal properties of rain and a fractal model, Tellus, 37 A, 209-232.

Lovejoy, S., Schertzer, D., Tsonis, A. A. (1987), Function box-counting and multiple elliptical dimension in rain, Science, 235, 1036-1038

Lovejoy, S., and D. Schertzer (1989), Comments on Are rain rate processes self-similar?

Water Resour. Res., 25, 577-579.

Lovejoy, S., Duncan, M.R., Schertzer, D. (1996), Scalar multifractal radar observer's problem, J. Geophys. Res., 101, 26479-26492.

Lovejoy, S., Schertzer, D. and Allaire, V., (2008). The remarkable wide range spatial scaling of TRMM precipitation, J. Atmos. Research, 90: 10-32

Macor, A. (2007), Développement de techniques de prévision de pluie basées sur les propriétés multi-échelles des données radar et satellites. Thèse de doctorat, Ecole Nationale des Ponts et Chaussées

Macor, J., Schertzer, D., Lovejoy, S. (2007), Multifractal Methods Applied to Rain Forecast Using Radar Data, La Houille Blanche, 4, 92-98.

Mandelbrot, B. (1989), Fractal geometry: what is it and what does it do? in Fractals in the Natural Sciences, edited by D. J. T. M. Fleischman, R. C. Ball, pp. 3-16, Princeton University press., Princeton.

Marsan, D., Schertzer, D., Lovejoy, S. (1996), Causal space-time multifractal processes: Predictability and forecasting of rain fields, J. Geophys. Res., 101, 26,333-326,346.

Marshall, J. S., and W. M. Palmer (1948), The distribution of raindrops with size, J. Meteor., $5,165-166$.

Nykanen, D.K. (2008), Linkages between Orographic Forcing and the Scaling Properties of Convective Rainfall in Mountainous Regions, J. of hydrometeorology 9, 327-347

Olsson, J., and J. Niemczynowicz (1996), Multifractal analysis of daily spatial rainfall distributions, J. of Hydrol., 187, 29-43.

Olsson, J. , Niemczynowicz, J. and Berndtsson, R. (1993), Fractal analysis of high-resolution rainfall time series. J.. Geophys. Res. 98 , pp. 23265-23274.

Parent du Châtelet, J., Tabary, P., Lamargue, P. (2005), Evolution du réseau radar opérationnel de Météo-France pour une meilleure estimation des lames d'eau, Hydrologie continentale 49

Parisi, G., Frish, U. (1985), A multifractal model of intermittency, dans Turbulence and predictability in geophysical fluid dynamics. North Holland, Ghill, M., R. Benzi, and G. Parisi, pp 111-114 
Radkevich, A., S. Lovejoy, K. Strawbridge, D. Schertzer, M. Lilley, 2008: Scaling turbulent atmospheric stratification, Part III: Space-time stratification of passive scalars from lidar data, Quart. J. Roy. Meteor. Soc., 134, 316-335.

Rodriguez-Iturbe, I., Gupta, V.K., Waymire, E. (1984), Scale considerations in the modelling of temporal rainfall, Wat. Resour. Res., 20, 1611-1619.

Royer, J.F., Biaou, A., Chauvin, F., Schertzer, Lovejoy, S. (2008), Multifractal analysis of the evolution of simulated precipitation over France in a climate scenario, C.R Geoscience 340, $431-440$

Schertzer, D., Lovejoy, S. (1987a), Physical modelling and analysis of rain and clouds by anisotropic scaling and multiplicative processes, J. Geophys. Res. 92(D8) : 9693-9714

Schertzer, D., Lovejoy, S. (1987b), Singularités anisotropes, divergences des moments en turbulence: invariance d'échelle généralisée et processus multiplicatifs, Ann. Sci. Math. Que, $11(1): 139-181$

Schertzer, D., Lovejoy, S. (1989b), Nolinear variability in geophysics multifractal analysis and simulations, dans Fractals Physical Origin and properties, L. Pietronero (Editeurs). Plenum Press, New York, pp 41-82

Schertzer, D., and S. Lovejoy (1992), Hard and soft multifractal processes, Physica A, 185(14), 187-194

Schertzer, D., and S. Lovejoy (1997), Universal Multifractals do Exist! J. Appl. Meteor., 36, 1296-1303

Schertzer, D., Tchiguirinskaia, I., Lovejoy, S., Hubert, P., Bendjoudi, H., \& Larchevêque, M. (2002), Which chaos in the rain-runoff process? J. Hydrological Sciences, 47, 139-148.

Schmitt, F., D. Lavallée, D. Schertzer and S. Lovejoy (1992). "Empirical Determination of Universal Multifractal Exponents in Turbulent Velocity Fields." Physical Review Letter 68: 305-308.

Solomon, S., D. Qin, M. Manning, Z. Chen, M. Marquis, K.B. Averyt, M. Tignor and H.L. Miller (eds.), Contribution of Working Group I to the Fourth Assessment Report of the Intergovernmental Panel on Climate Change, Cambridge University Press, Cambridge, United Kingdom and New York, NY, USA, 2007996 pp

Stolle, J., S. Lovejoy, D. Schertzer (2009), The stochastic cascade structure of deterministic numerical models of the atmosphere, Nonlinear Proc. in Geophys., 16, 1-15

Tessier, Y., S. Lovejoy, D. Schertzer (1993), Universal Multifractals: theory and observations for rain and clouds, J. Appl. Meteor., 32, 223-250.

Tchiguirinskaia, I., Schertzer, D., Hubert, P., Bendjoudi, H., Lovejoy, S. (2004), Multiscaling geophysics and sustainable development, Scales in Hydrology and Water Management, IAHS Publ. 287, 113-136 
Testud, J., Le Bouar, E., Obligis, E., and AliMehenni, M. (2000), The rain profiling algorithm applied to polarimetric weather radar. J. Atmos. Oceanic Tech., 17, 332-356

Veneziano, D, and Furcolo, P., (1999), A modified double trace moment method of multifractal analysis, Fractals, 7, 181-195

Waymire, E., and V. K. Gupta (1981), The mathematical structure of rainfall representations, 3, Some applications of the point process theory to rainfall processes, Wat. Resour. Res., 17, 1287-1294.

Yaglom, A. M. (1966). "The influence on the fluctuation in energy dissipation on the shape of turbulent characteristics in the inertial interval." Sov. Phys. Dokl. 2: 26-30.

Zawadzki, I., 1987, Fractal versus correlation structure in rain, Journal of Geophysical Research, 92, 469-472

\begin{tabular}{|c|c|c|c|c|c|c|c|c|c|c|c|c|}
\hline & \multicolumn{4}{|c|}{ Grandes échelles } & \multicolumn{5}{c|}{ Petites échelles } \\
\cline { 2 - 15 } & \multicolumn{3}{|c|}{ Méso-NH } & \multicolumn{3}{c|}{ Radar } & \multicolumn{3}{c|}{ Méso-NH } & \multicolumn{3}{c|}{ Radar } \\
\cline { 2 - 14 } & $\alpha$ & $C_{1}$ & $H$ & $\alpha$ & $C_{1}$ & $H$ & $\alpha$ & $C_{1}$ & $H$ & $\alpha$ & $C_{1}$ & $H$ \\
\hline Moyenne & 0.61 & 0.78 & 0.37 & 0.78 & 0.51 & 0.34 & 1.12 & 0.17 & 0.70 & 1.56 & 0.17 & 0.51 \\
\hline Ecart-type & 0.18 & 0.20 & 0.15 & 0.17 & 0.12 & 0.13 & 0.22 & 0.06 & 0.14 & 0.29 & 0.07 & 0.12 \\
\hline $\begin{array}{c}\text { Coeff. de } \\
\text { variation } \\
(\%)\end{array}$ & 29 & 26 & 40 & 22 & 24 & 38 & 19 & 38 & 20 & 18 & 43 & 24 \\
\hline
\end{tabular}

Tableau 1 : Statistiques des paramètres $\alpha, C_{1}$, et $H$ pour les données radar et les simulations Méso-NH en analyse spatiale par échantillon

\begin{tabular}{|c|c|c|c|c|c|c|c|c|}
\hline & \multicolumn{4}{|c|}{ Méso-NH } & \multicolumn{4}{c|}{ Radar } \\
\cline { 2 - 10 } & $\alpha$ & $C_{1}$ & $H$ & $\gamma_{s}$ & $\alpha$ & $C_{1}$ & $H$ & $\gamma_{s}$ \\
\hline Grandes échelles & 0.54 & 0.71 & 0.36 & 1.20 & 0.89 & 0.45 & 0.34 & 1.06 \\
\hline Petites échelles & 1.20 & 0.14 & 0.58 & 0.61 & 1.62 & 0.16 & 0.59 & 0.84 \\
\hline
\end{tabular}

Tableau 2: Paramètres $\alpha, C_{1}, H$ et $\gamma_{s}$ pour les données radar et les simulations Méso-NH, en analyse spatiale, en faisant des moyennes d'ensemble sur les différents pas de temps 


\begin{tabular}{|c|c|c|c|c|}
\hline \multirow{2}{*}{} & \multicolumn{2}{|c|}{ Méso-NH } & \multicolumn{2}{c|}{ Radar } \\
\cline { 2 - 5 } & $\alpha$ & $C_{1}$ & $\alpha$ & $C_{1}$ \\
\hline Moyenne & 0.49 & 0.56 & 0.57 & 0.41 \\
\hline Ecart-type & 0.29 & 0.19 & 0.26 & 0.17 \\
\hline $\begin{array}{c}\text { Coeff. de } \\
\text { variation (\%) }\end{array}$ & 59 & 34 & 45 & 41 \\
\hline Corr. avec $C_{F}$ & -0.86 & 0.85 & -0.78 & 0.83 \\
\hline
\end{tabular}

Tableau 3 : Statistiques des paramètres $\alpha$ et $C_{1}$ pour les données radar et Méso-NH pour les la dernière période de simulation en analyse temporelle échantillon par échantillon.

\begin{tabular}{|c|c|c|c|c|c|c|}
\hline & \multicolumn{3}{|c|}{ Méso-NH } & \multicolumn{3}{c|}{ Radar } \\
\cline { 2 - 7 } & $\alpha$ & $C_{1}$ & $H$ & $\alpha$ & $C_{1}$ & $H$ \\
\hline Grandes échelles & 0.54 & 0.50 & 0.37 & 0.82 & 0.35 & 0.21 \\
\hline Petites échelles & 0.73 & 0.30 & 0.49 & 1.16 & 0.26 & -0.01 \\
\hline
\end{tabular}

Tableau 4: Statistiques des paramètres $\alpha$ et $C_{1}$ pour les données radar et les simulations Méso-NH en analyse temporelle et en moyenne d'ensemble

\begin{tabular}{|c|c|c|c|c|}
\hline \multirow{2}{*}{} & \multicolumn{2}{|c|}{ Méso-NH } & \multicolumn{2}{c|}{ Radar } \\
\cline { 2 - 5 } & $\alpha$ & $C_{1}$ & $\alpha$ & $C_{1}$ \\
\hline Grandes échelles & 0.68 & 0.47 & 0.87 & 0.42 \\
\hline Petites échelles & 1.07 & 0.21 & 1.43 & 0.23 \\
\hline
\end{tabular}

Tableau 5 : Paramètres $\alpha$ et $C_{1}$ pour les données radar et les simulations Méso-NH, en analyse spatio-temporelle, en faisant des moyennes d'ensemble.

\section{LEGENDE DES FIGURES}




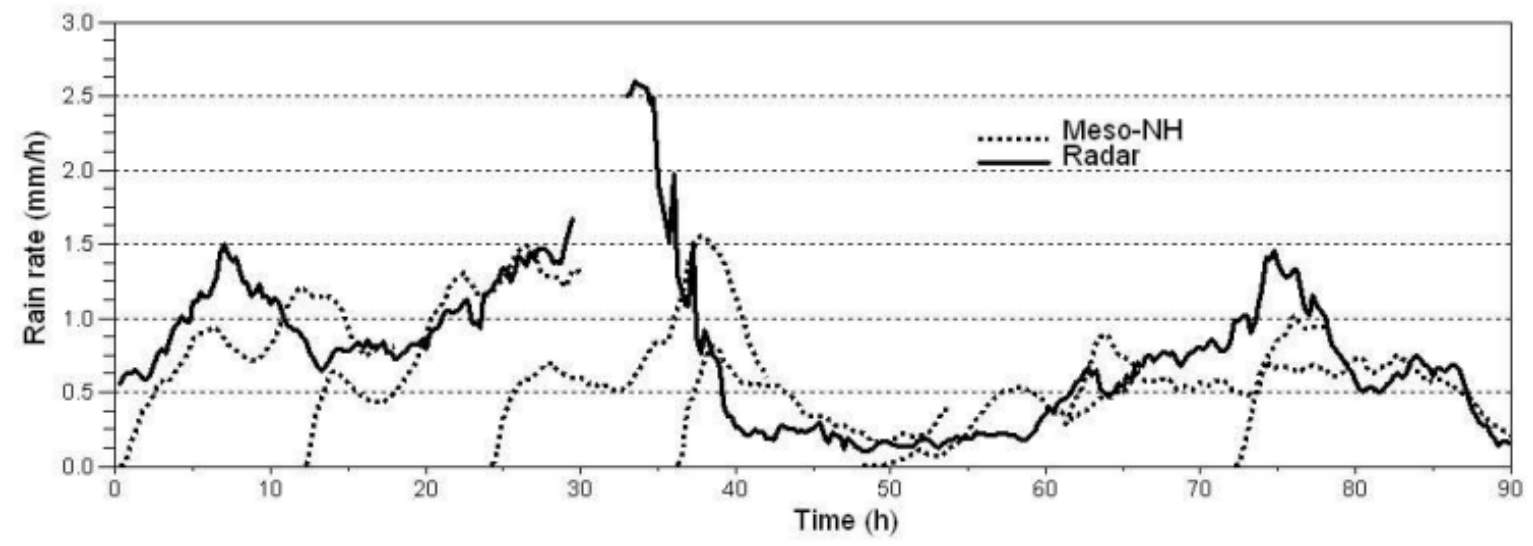

Figure 1 : Evolution de l'intensité moyenne des précipitations sur la zone étudiée au cours de l'évènement étudié pour les deux types de données : simulation Méso-NH (rouge) et données $\operatorname{radar}($ noir)
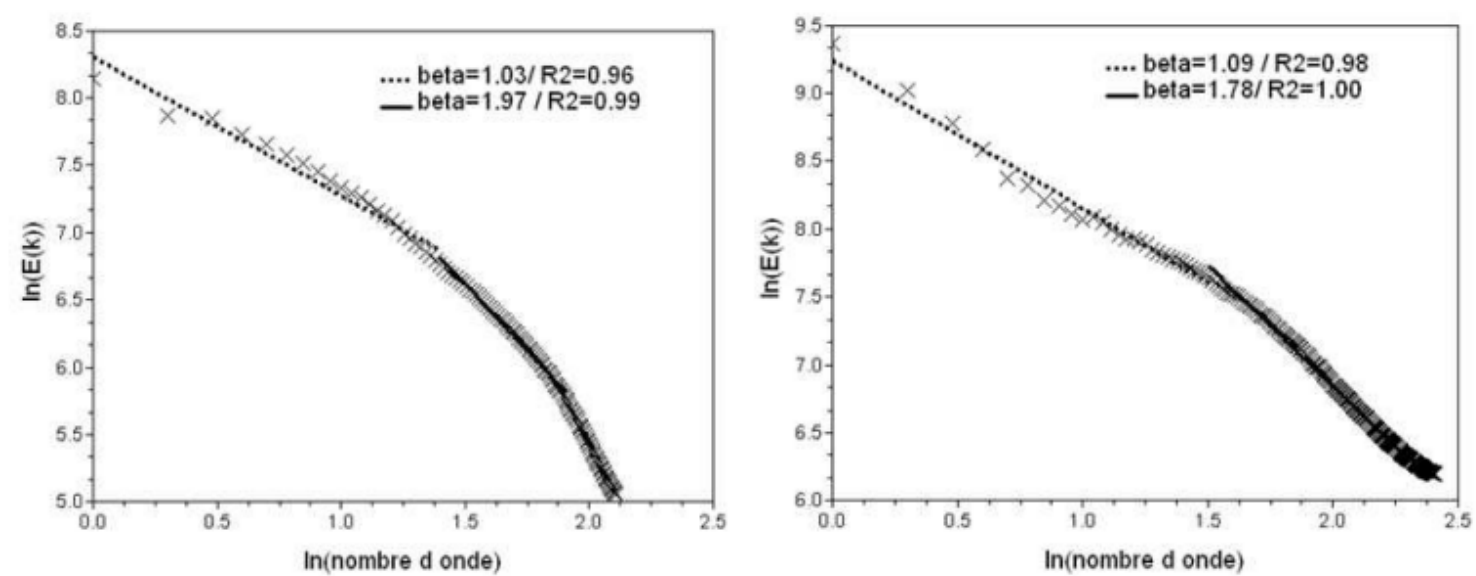

Figure 2 : Moyenne d'ensemble des spectres en analyse spatiale : Méso-NH (à droite) et radar (à gauche)
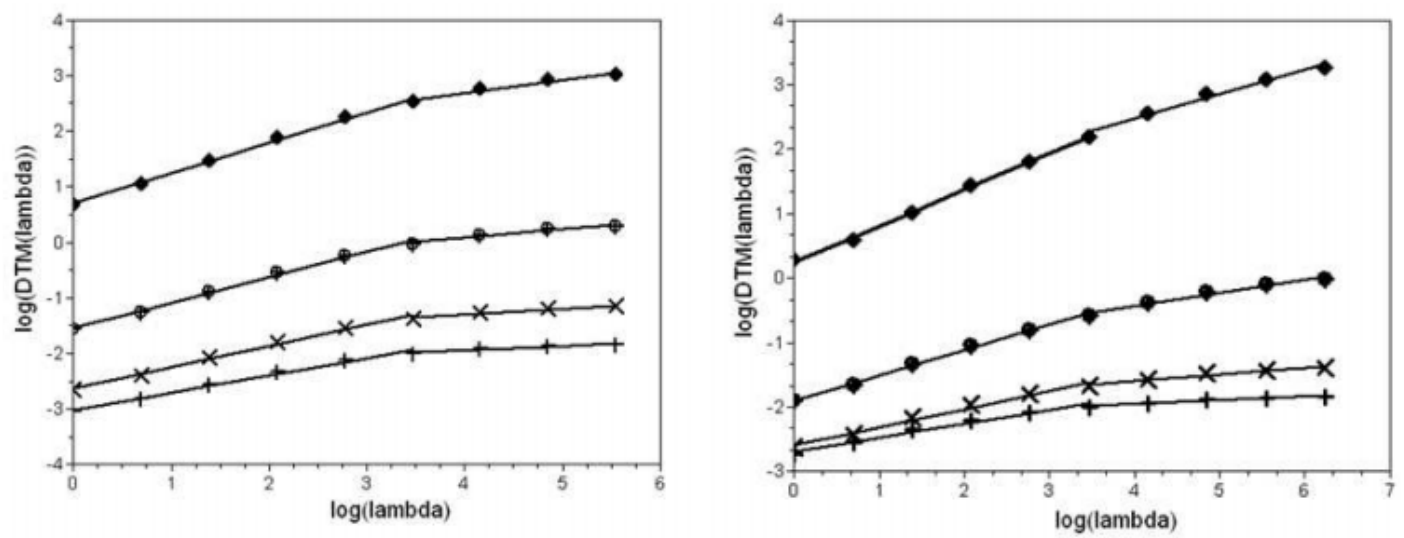
Figure 3 : DTM $(q=1.5, \eta=0.66 ; 1 ; 1.52 ; 2.31)$ en fonction de la résolution en graphique log$\log$ pour l'analyse spatiale en moyenne d'ensemble. (à gauche) Simulation Méso-NH (à droite) Données radar. Les coefficients de régression linéaire $R 2$ pour les grandes échelles, pour les petites échelles et sans prendre en compte de rupture valent respectivement 0.99, 0.96 et 0.95 pour les données Méso-NH et $0.99,0.96$ et 0.95 pour les données radar.
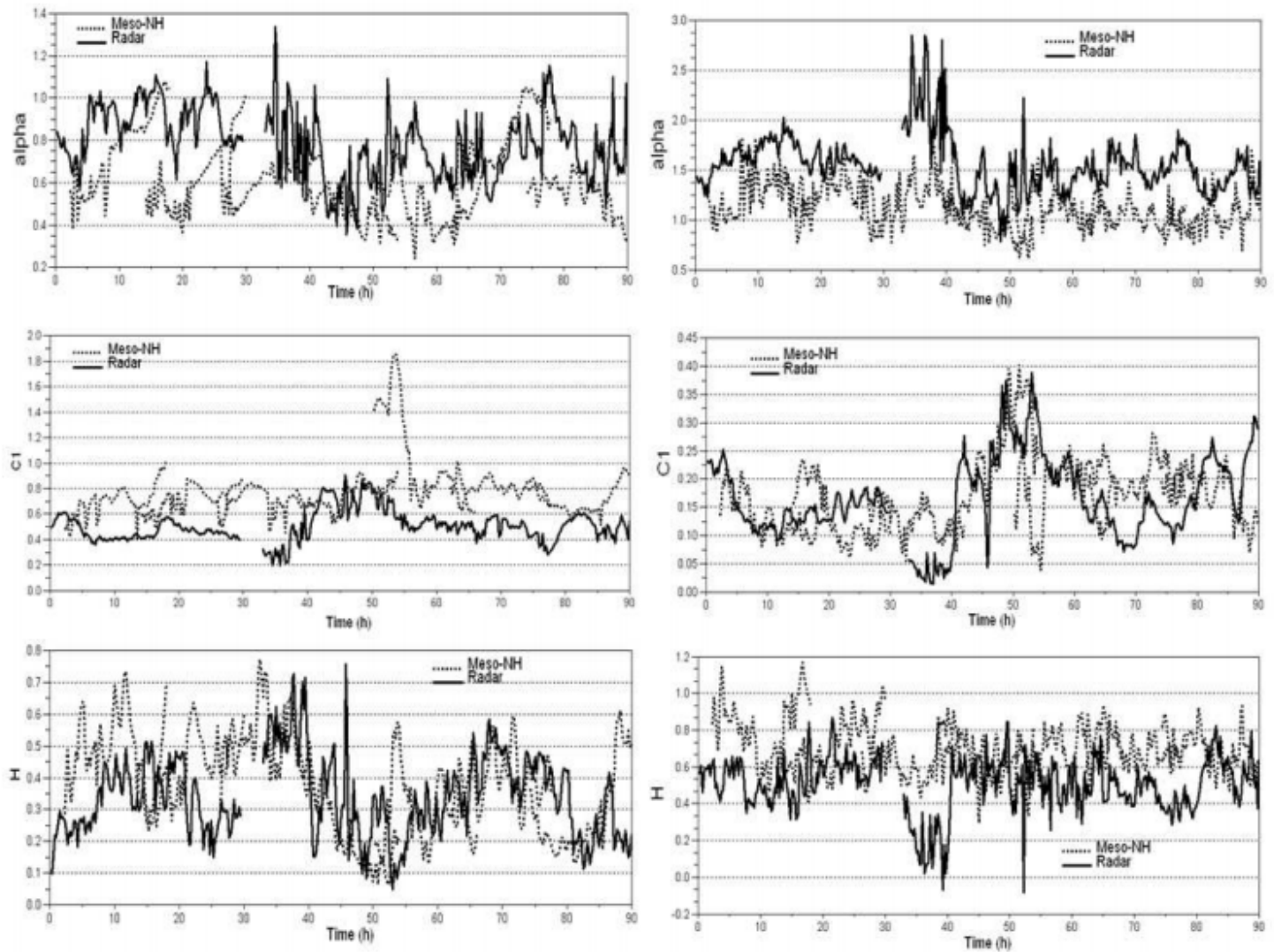

Figure 4 : Evolution temporelle des paramètres $\alpha, C_{1}$ et $H$ pour les données radar et les simulations Méso-NH, en analyse spatiale par échantillon. Grandes échelles (à gauche) et petites échelles (à droite). 

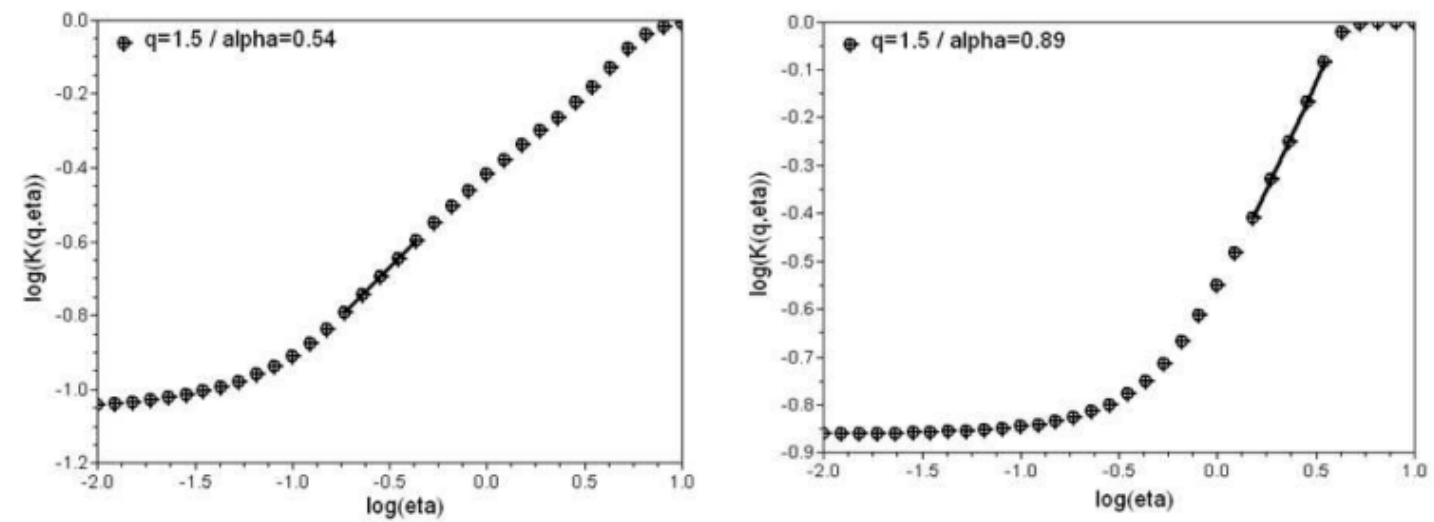

Figure 5 : Courbes de détermination de $\alpha$ dans la méthode DTM pour l'analyse spatiale en moyenne d'ensemble (sur les différents pas de temps). Méso-NH (à gauche) et Radar (à droite)
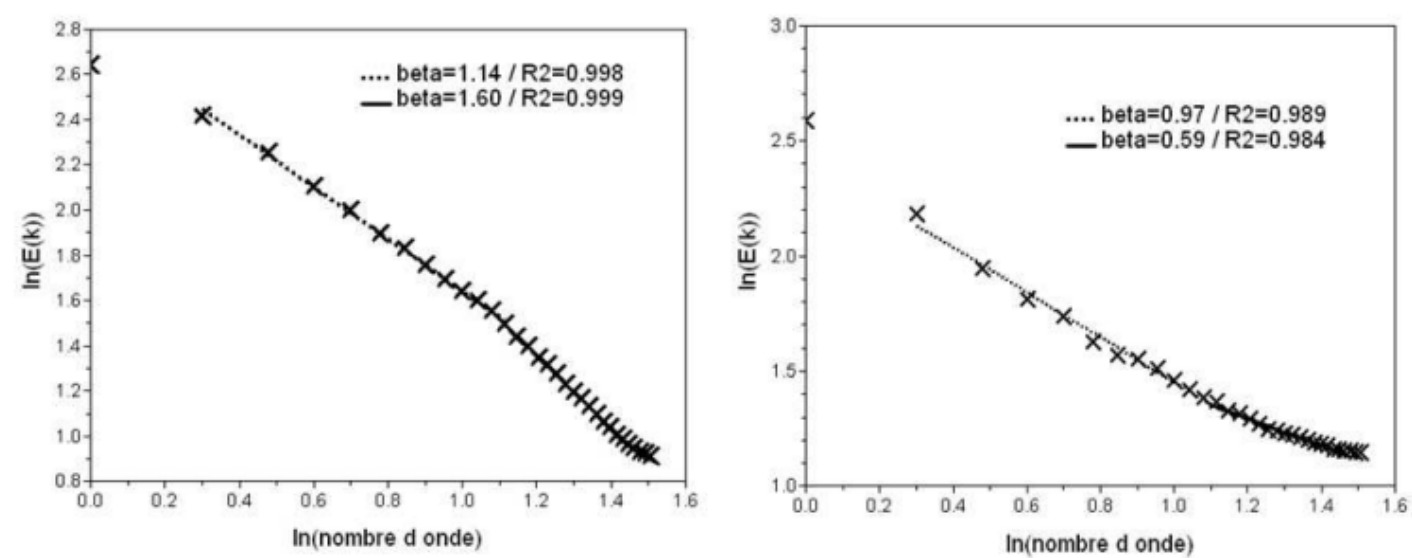

Figure 6 : Moyenne d'ensemble des spectres en analyse temporelle : Méso-NH (à droite) et radar (à gauche) 

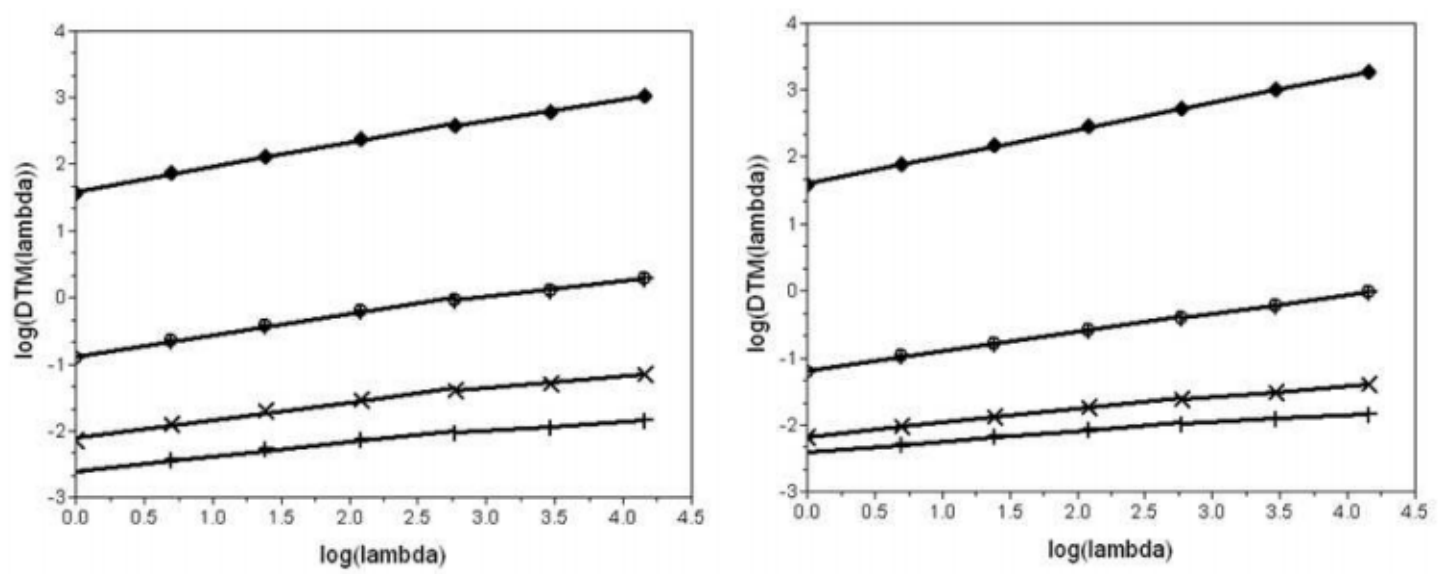

Figure 7 : DTM $(q=1.5, \eta=0.66 ; 1 ; 1.52 ; 2.31)$ en fonction de la résolution en graphique loglog pour l'analyse temporelle en moyenne d'ensemble. (à gauche) Simulation Méso-NH (à droite) Données radar. Les coefficients de régression linéaire $R 2$ pour les grandes échelles, pour les petites échelles et sans prendre en compte de rupture valent respectivement 0.99, 0.99 et 0.98 pour les données Méso-NH et $0.99,0.99$ et 0.99 pour les données radar. 

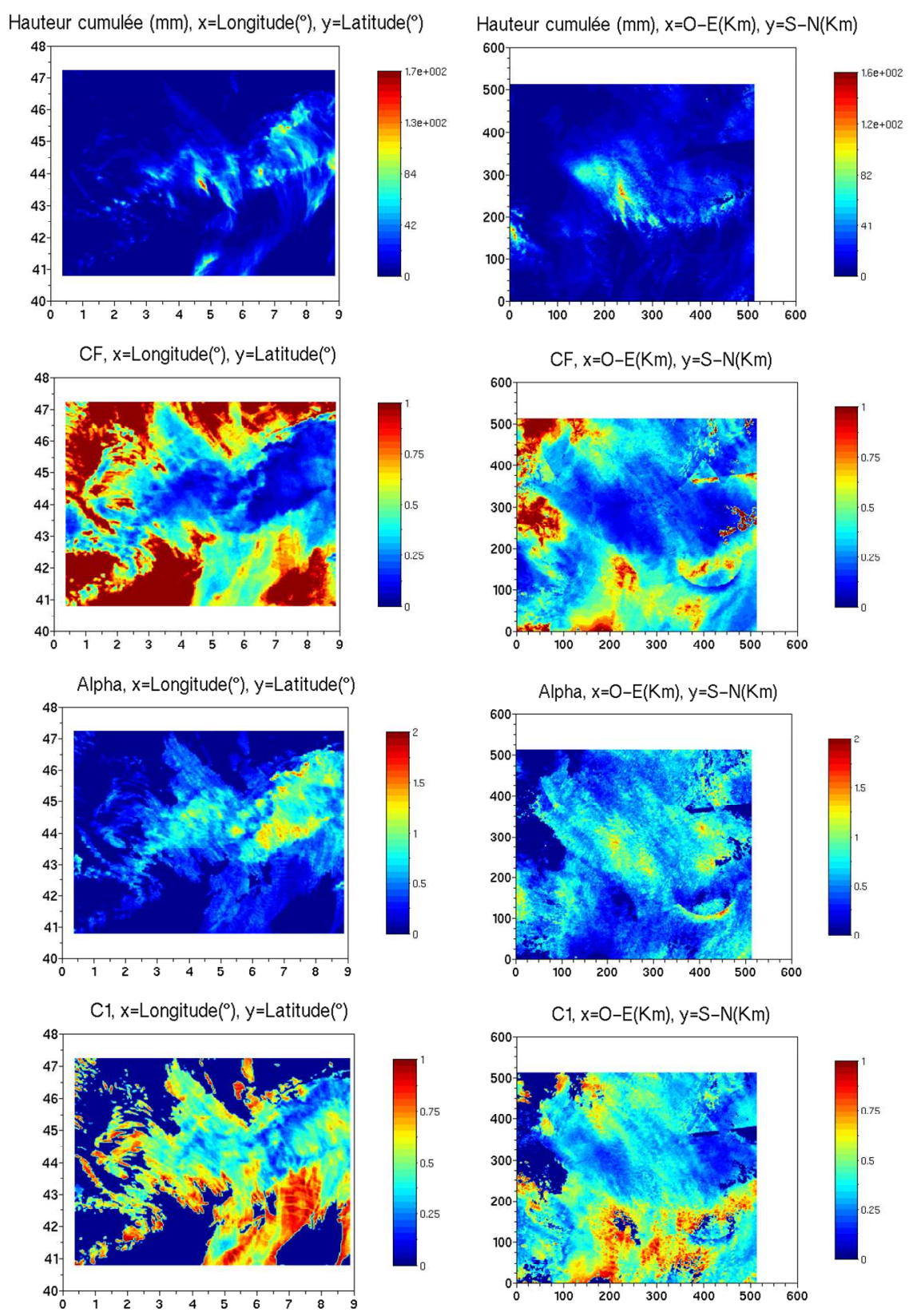

Figure 8: Cartes d'analyse temporelle pour les pas de temps correspondant à la dernière période de simulation Méso-NH : simulation Méso-NH (à gauche) et données radar (à droite). Les coordonnées exprimées en latitude et longitude des 4 extrémités de l'image radar sont respectivement 46.3-1.3, 41.5-1.3, 46.2-8.1 et 41.4-7.5. 

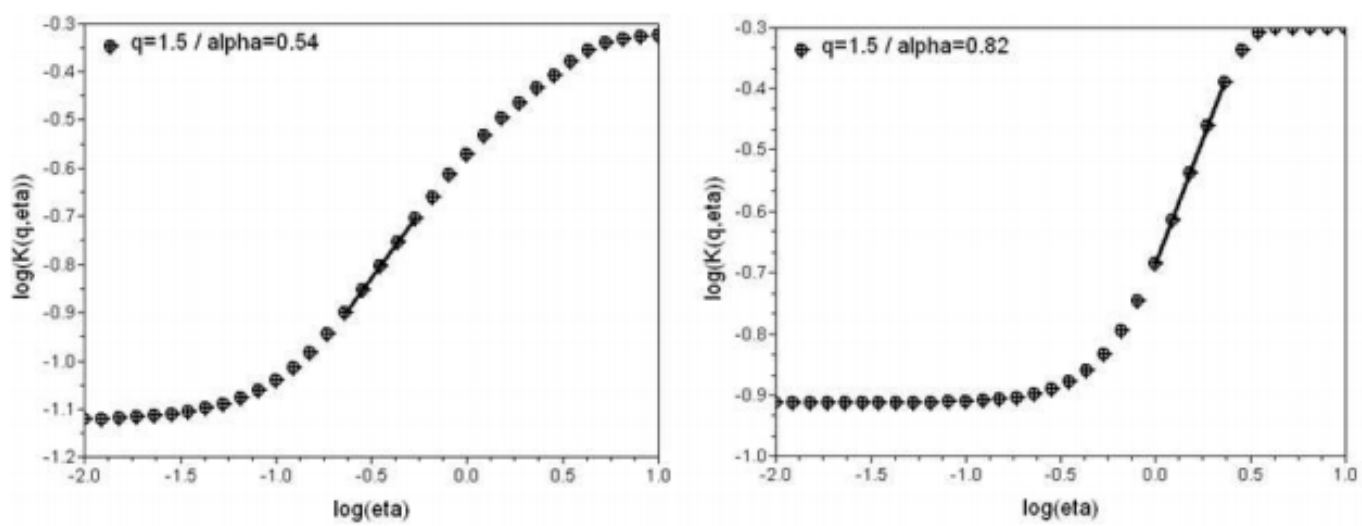

Figure 9 : Courbes de détermination de $\alpha$ dans la méthode DTM pour l'analyse temporelle en moyenne d'ensemble (sur les pixels des différentes simulations). Méso-NH (à gauche) et Radar (à droite)
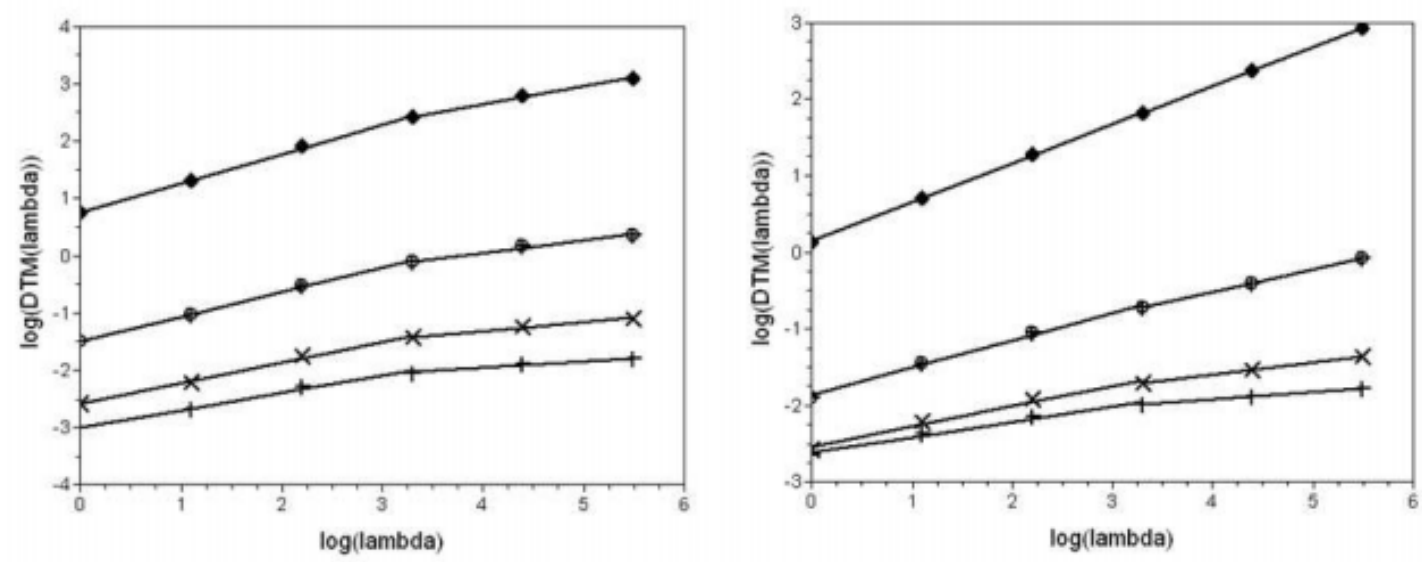

Figure 10 : DTM $(q=1.5, \eta=0.66 ; 1 ; 1.52 ; 2.31)$ en fonction de la résolution en graphique loglog pour l'analyse spatio-temporelle en moyenne d'ensemble. (à gauche) Simulation MésoNH (à droite) Données radar. Les coefficients de régression linéaire $R 2$ pour les grandes échelles, pour les petites échelles et sans prendre en compte de rupture valent respectivement 0.99, 0.98 et 0.97 pour les données Méso-NH et $0.99,0.99$ et 0.98 pour les données radar. 

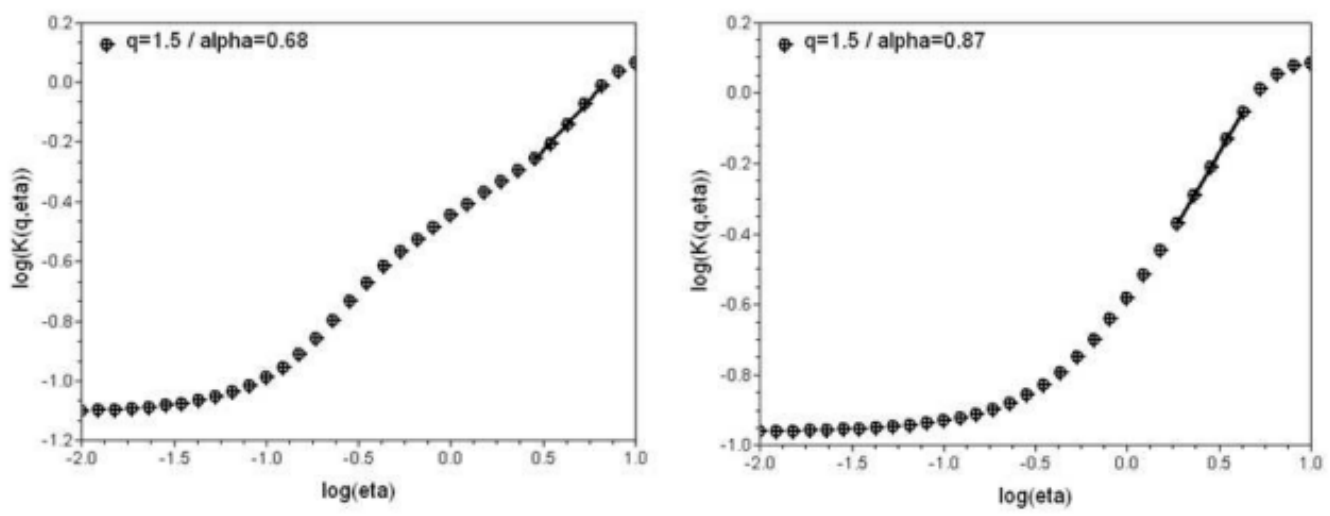

Figure 11: Courbes de détermination de $\alpha$ dans la méthode DTM pour l'analyse spatiotemporelle en moyenne d'ensemble. Méso-NH (à gauche) et Radar (à droite). 